\title{
Hemolytic uremic syndrome
}

\author{
Iliyan Todorov ${ }^{1}$, Dimitrichka Bliznakova ${ }^{2}$, Margarita \\ Gospodinova'.
}

\author{
1. Department of Infectious Diseases, Parasitology \\ and Dermatology, Medical University of Varna;
}
2. Department of Pediatrics and Medical Genetics, Medical University of Varna;

\begin{abstract}
Hemolytic uremic syndrome is a form of thrombotic microangiopathy which takes its clinical course by a triad of symptoms: microangiopathic hemolytic anemia, thrombocytopenia and acute renal failure. We review a considerable amount of literature about the mentioned problem. Two kinds of hemolytic uremic syndrome are known today: the first one is associated with a preceding diarrheal episode, most commonly caused by Escherichia coli, while the second one is not associated with diarrhea, but with different streptococcal infections or genetic mutations. Because antibiotics and anti-diarrheal drugs play a role as a trigger factor for the disease, supportive care is the essential treatment of patients with diarrhea-associated variant of the disease and plasma therapy for nondiarrheal hemolytic uremic syndrome. New therapeutic strategies are developed such as SYNSORB for diarrheal hemolytic uremic syndrome and Eculizumab for the non-diarrheal variant. The prognosis of the disease is auspicious, but what about non-diarrheal forms, up to $25 \%$ of patients die in the acute phase and $50 \%$ progress to end stage renal disease /ESRD/. For these reasons the characteristics of hemolytic uremic syndrome have to be known in details and physicians have to focus on early diagnosis and treatment of the disease when its prognosis stays auspicious.
\end{abstract}

Keywords: Hemolytic uremic syndrome, diarrhea, Shiga like toxin, E. coli, ADAMTS13, thrombotic thrombocytopenic purpura

\section{Background}

Hemolytic uremic syndrome /HUS/ is a clinical syndrome associated with a triad of symptoms: acute kidney failure, microangiopathic hemolytic anemia /MHA/ and thrombocytopenia. HUS was first described by Conrad von Gasser et al. in 1955 in 5 children with the above-mentioned symptoms. As a consequence, the disease is also known as Gasser syndrome $(1,2,3)$. Thirty years later, Mohamed A. 
Karmali et al. found the cause of the disease: all patients have had an E. coli strain in their feces $(4,6)$. Two groups of HUS are known today (see Table 1). More than $80-90 \%$ of the cases are included in the group of ,typical” or diarrhea-associated or Shiga toxin-associated HUS /tHUS; D+HUS; Stx-HUS/, which affects children mainly, begins after a period of acute diarrhea, develops as a sporadic disease or in epidemics and its prognosis is auspicious $(1,2,3,5,8,9,10,11,13)$. ,Atypical” HUS or diarrhea-unrelated HUS /aHUS, D-HUS; non-Stx-HUS/ accounts for $10 \%$ of all HUS cases and may be sporadic or familial. Atypical HUS is often associated with pneumococcal infections such as meningitis, pneumonia or sepsis, autoimmune diseases, HIV, transplantation, etc.; its clinical course is more severe than that of tHUS; it often stays resistant to treatment, with frequent relapses and chronic progression to an end stage renal disease /ESRD/ $(1,2,3,4,5,8,9,10,11,13,14,15,17,25)$.

\begin{tabular}{|c|c|}
\hline \multirow{2}{*}{$\begin{array}{l}\text { Types of HUS } \\
\text { Typical HUS }\end{array}$} & Cause \\
\hline & Verotoxin-producing E. coli \\
\hline & Shigella disenteriae \\
\hline \multicolumn{2}{|l|}{ Atypical HUS } \\
\hline - Infectious related HUS & $\begin{array}{l}\text { Str. pneumoniae } \\
\text { Salmonella typhi } \\
\text { Campylobacter jejuni } \\
\text { Neisseria meningitides } \\
\text { Mycoplasma sp. } \\
\text { Legionella pneumophilia } \\
\text { Yersinia pseudotuberculosis } \\
\text { HIV** } \\
\text { Enteroviruses } \\
\text { Influenza virus } \\
\text { EBV** } \\
\text { HSV*** }_{\text {Aspergillus fumigates }}\end{array}$ \\
\hline $\begin{array}{l}\text { - Genetic variants of HUS } \\
\checkmark \quad \text { Complement factor } \\
\text { abnormalities }\end{array}$ & $\begin{array}{l}\text { Factor } \mathrm{H} \text { deficiency } \\
\text { Factor I deficiency } \\
\text { Membrane cofactor protein } \\
\text { deficiency } \\
\text { Factor B excessive activity } \\
\text { Complement } 3 \text { excessive } \\
\text { activity }\end{array}$ \\
\hline$\checkmark \begin{array}{l}\text { ADAMTS 13 } \\
\text { deficiency }\end{array}$ & Genetic mutation \\
\hline $\begin{array}{l}\text { Cobalamin metabolism } \\
\text { defect }\end{array}$ & Genetic mutation \\
\hline \multirow[t]{7}{*}{$\checkmark \quad$ Miscellaneous } & Connective tissue disease \\
\hline & Organ transplantation \\
\hline & Drugs \\
\hline & Malignancy \\
\hline & Radiation \\
\hline & Pregnancy \\
\hline & Idiopathic \\
\hline
\end{tabular}

${ }^{*} \mathrm{HIV}$ : Human immunodeficiency virus; ${ }^{* \star} \mathrm{EBV}$ : Epstein-Barr virus; ${ }^{* \star} \mathrm{HSV}$ : Herpes simplex virus; ${ }^{* * * *}$ ADAMTS-13: a disintegrin-like and metalloprotease with thrombospondin type 1 repeats, number 13 
During childhood more than $90 \%$ of HUS cases follow an episode of acute diarrhea /tHUS;

$\mathrm{D}+\mathrm{HUS} /(1,2,3,4,5,8,9,11,13)$. The most common cause /up to $83 \% /$ of $\mathrm{D}+\mathrm{HUS}$ worldwide is infection by Shiga like-toxin/verotoxin producing enterohemorrhagic strain of Escherichia coli /EHEC/, mainly 0157:H7 $180-86 \% /$, but only $10-15 \%$ of infected patients will develop HUS. Other serotypes that are associated with HUS are 0111:H8, 0103:H2, 0121, 0145, 026, 0113, 0104:H4. $(1,3,4,6,8,9,10,11,13,21,22,28)$. The risk for HUS occurrence increases when patients are treated with loperamids, using amoxicillin alone or in combination with gentamicin, trimethoprim-sulfamethoxazol (16). Less frequently observed agents are Shigella dysenteriae type 1 or Citrobacter freundii. E. coli urinary tract infections cause this clinical syndrome rarely (6).

D-HUS affects all age groups, but adolescents and adults predominantly, without previous diarrheal episodes and accounts for $5-10 \%$ of all cases worldwide. It occurs sporadically or in families $(1,5,7,14,21,22)$.

Different risk factors and diseases are known as conducive to sporadic D-HUS development (see Table 1). In more than $40 \%$ of cases it starts in the course of a Streptococcus pneumoniae infection, such as respiratory tract infections - pneumonia, empyema, mastoiditis, otitis media or other streptococcal diseases for example meningitis, pericarditis, peritonitis, sepsis. Pneumococcal serotypes reported to cause HUS are 1, 2F, 3, 6A, 6B, 8, 9V, 14, 19 and 23F (24,36,37). On the surface of renal endothelial cells, platelets, red blood cells and hepatocytes, a specific antigen, called Thomsen-Friedenreich antigen /TAg/, is located, which is normally inhibited by neuraminic acid. Streptococcus pneumoniae produces a circulating neuraminidase that removes neuraminic acid from the surface of the mentioned cells, leading to a reaction between TAg and anti-TAg antibodies. Anti-TAg antibodies circulate normally in the serum after 6 months of age. After binding, an immune cascade becomes activated resulting in hemolytic anemia, thrombocytopenia, vascular injury and hepatic dysfunction $(1,3,6,8,9,36,37)$.

Among the numerous other microorganisms that are associated with sporadic D-HUS are salmonella typhi, campylobacter jejuni, yersinia pseudotuberculosis, neisseria meningitidis, legionella pneumophilia, mycoplasma species, rickettsia, HIV, enteroviruses, influenza virus, Epstein-barr virus and herpes simplex virus. Aspergillus fumigates is a rare etiologic agent. Sometimes HUS occurs as a consequence of vaccination with an influenza triple antigen vaccine, typhoid-paratyphoid $A$ and $B$ vaccine, or polio vaccine $(1,3,4,8,10,21,22,23,28,34,35)$.

Sporadic D-HUS during pregnancy is a complication of different conditions such as preeclampsia, eclampsia, abortion, etc. Its prognosis stays auspicious and becomes better after delivery. Postpartum HUS appears within 3 months of delivery as a result of abruptio placentae, amniotic embolia or puerperal sepsis. It is associated with severe changes in the kidneys and its mortality rate is about $50-60 \%$ with residual renal dysfunction and malignant hypertension $(5,8)$.

A wide group of drugs are associated with sporadic D-HUS development whose symptoms may start 1 year after the beginning of the treatment. Four categories of medicaments are important as triggers: anticancer drugs (cisplatin, gemcitabine, bleomycin, mitomycin), immunomodulators such as cyclosporine, tacrolimus, interferon, quinidine; antiplatelet (clopidogrel; ticlopidine) and oral contraceptives. The prognosis is poor with a mortality rate of about $75 \%(1,3,21,22)$. 
According to different clinical trials, sporadic D-HUS may reoccur in the transplanted kidney (posttransplantation HUS) in two variants: de novo posttransplantation HUS in patients who had never experienced the disease before and had been treated with immune drugs as cyclosporine $(5-15 \%)$, tacrolimus (1\%) or recurrent posttransplantation HUS (13\%), which affects persons with previous episodes of HUS leading to ESRD $(17,26,27,29,30)$.

Rarely some tumors, chiefly mucin producing adenocarcinomas, are mentioned as causative factors for sporadic D-HUS $(2,38)$.

Connective tissue disorders are identified as risk factors for sporadic D-HUS development. The most important diseases are lupus erithematodes, scleroderma and antiphospholipid antibody syndrome. Primary glomerulopathies and allogenic hematopoetic cell transplantation account for about $0.05 \%$ of all HUS cases $(15,16,35)$.

No cause is identified in about $50 \%$ of all cases of sporadic D-HUS $(11,13,28)$.

Familial D-HUS accounts for $3 \%$ of all cases of HUS (25). Autosomal dominant inheritance (AD) and autosomal recessive inheritance (AR) are known today. It affects both children and adults (AR more often children), with poor prognosis and a mortality rate of $60-70 \%$ (2). The reason for that hereditary disorder is unknown, but some suggestions include congenital defects of complement system collagen type III glomerulopathy, prostacyclin exchange disturbances and cobalamine metabolism defects with methylmalonic aciduria and homocystinuria $(14,15,25,33,38)$.

Familial D-HUS as a result of complement abnormalities affects more often children and specific mutations are identified in 30-50\% (14). This includes (see Table 2):

- factor H deficiency (14-33\%) - a glycoprotein which plays an important role in the regulation of alternative pathways of complement. It blocks C3 convertase and acts as a factor I cofactor resulting in $\mathrm{C} 3 \mathrm{~b}$ proteolysis. More than 100 mutations of factor $\mathrm{H}$ are known today, separated into two groups: quantitative deficit (type 1) - leading to low C3 levels in plasma; functional deficit (type 2) - with normal levels of the mentioned protein. That form of D-HUS presents during infancy or early childhood $(5,7,14,25,38)$.

- Factor I deficiency (2-13\%) - more than 40 kinds of factor I mutations have been identified. All of them are heterozygous. The sequences include defects in protein secretion or changes in the protein structure, which leads to reduction in its co-factorial activity $(7,14,25)$.

- Membrane cofactor protein deficiency (3-17\%) - the mutant membrane cofactor protein /MCP/ cannot bind to C3b and its ability as a co-factor becomes low. Homozygous and heterozygous kinds of mutations are identified predominantly in children, rather than in adults $(5,14)$.

- Factor B excessive activity (0-5\%) - this kind of mutation is rare, heterozygous and leads to increased stability and activity for $\mathrm{C} 3$ convertase which supports the alternative pathway of the complement system even in very low concentrations $(5,14)$. 
- Complement 3 excessive activity (2-17\%) - it is a heterozygous mutation and C3 binds weakly to MCP causing a gain of function. As a result, there is an increased binding of complement factor $B$ to C3b and an increase in C3 convertase $(5,14)$.

- Combined mutation (12\%) - a presence of two or more of the abovementioned mutations $(5,14)$.

Table 2 Gene mutation rate of different complement factors, Salvadori M et al., Hemolytic uremic syndrome: an update, 2013

\begin{tabular}{|c|c|c|}
\hline Abnormality & Gene locus & aHUS rate \\
\hline Factor H & CFH (RCA:1q32) & $11-29 \%$ \\
\hline Membrane co-factor protein & MCP (RCA) & $3-17 \%$ \\
\hline Factor I & CFI (4q25) & $2-17 \%$ \\
\hline C3 & C3 (19p13) & $2-17 \%$ \\
\hline Factor B & CFB (6p21) & $0-5 \%$ \\
\hline Thrombomodulin & THBD (20p11) & $0-5 \%$ \\
\hline
\end{tabular}

Another cause for Familial D-HUS may be some mutations in the ADAMTS13 gene (a disintegrin-like and metalloprotease with thrombospondin type 1 repeats, number 13), located on chromosome 9q34, which is a hereditary disorder or acquired. The AR inheritance of that mutation affects children mainly and accounts for $5 \%$ of familial D-HUS. Acquired forms are presented by the appearance of anti-ADAMTS 13 antibodies and affects mainly adults or older children. This variant of D-HUS is related to trhrombotic thrombocytopenic purpura (TTP) and causes central nervous system involvement more often than kidneys'. ADAMTS13 is an enzyme produced by a special kind of cells in the liver. It acts as a von Willebrand factor (VWF) cleaving protease, and its deficiency is associated with the circulation in the plasma of the ultralarge multimeric form of VWF (ULVWF), which binds the platelets, leading to thrombocytopenia and embolisation of small vessels causing tissue ischemia $(18,19,23)$.

A rare variant of thrombotic microangiopathy (TMA) is TTP whose clinical signs and symptoms resemble the features of HUS. TTP affects adults primarily, involves central the nervous system more than the kidneys, and a high relapse rate is typical. TTP is the result of severe acquired ADAMTS 13 deficiency caused by anti-ADAMTS13 antibodies. In up to $5 \%$ of the cases, inherited TTP develops as a result of a mutation in the ADAMTS 13 gene $(7,18,19,23)$.

Some defects in vitamin B12 metabolism - homozygous or heterozygous mutations on chromosome 1p34 account for $25 \%$ of cases of familial D-HUS, predominantly in children before the age of 6 months. The inheritance of this defect is AR. It is associated with changes in the transformation of homocysteine into methionine and conversion of methylmalonyl - CoA to succinyl - CoA. The result is methylamonic acidemia and homocystinuria $(7,33)$.

The idiopathic AR form of HUS affects both children and adults and causes a fulminant progression to ESRD with a poor prognosis. Idiopathic AD is arare form of aHUS which affects members of one family at different ages. This form is also associated with a poor prognosis and the development of ESRD.

The D+HUS associated morbidity is about 2.1 per 100000 persons annually $(2,4,6,8)$. Children up to 5 years of age are more often affected - the frequency is 6.1 per 100000 . In the age group of $50-59$, the 
disease accounts for 0.5 cases per 100000 people annually $(9,10,13)$. The seasonality of $\mathrm{D}+\mathrm{HUS}$ follows the seasonal characteristic of the E. coli infection - during the summer between June and September. The disease is distributed in North America, West Europe, Japan, South America, Africa and Australia. In the United States, 70000 illnesses and 60 deaths are registered annually. In countries endemic for E. coli such as Argentina and Uruguay, D+HUS accounts for an incident rate of 10.5 per 100000 annually. In the developed countries, the morbidity associated with E. coli infections accounts for 1-30 cases per 100000 people. In the time of E. coli epidemics, $38-61 \%$ of infected persons develop bloody diarrhea, but only 2$8 \%$ of cases progress to HUS $(6,20,21,22,23)$.

In May 2011, a big epidemic of D+HUS caused by E. coli 0104:H4 was registered in Germany. It resulted from eating imported Spanish cucumbers, contaminated by E. coli (9). On 27th May, 2011 a big epidemiologic study was started including analyses of soil, water and biological material from suspected greenhouses in the endemic region of Andalusia. The samples were negative for E. coli O104:H4 and a new possibility for the cause of the contamination was supposed -cross-contamination of the product during its transport on the territory of Germany. The Federal Epidemiologic Centre in the Robert Koch Institute published a reminder to people to stop eating uncooked tomatoes, cucumbers and lettuce. In spite of this, German newspapers continued to accuse Spain as a guilty country for the beginning of that epidemic and different articles contributed the contamination of the cucumbers to their pouring over with contaminated water or conceived of it as transmitted by Spanish slugs Arion vulgaris, which are more ,,frequent problems for Spain than Germany". On 4th June, the focus was moved to a restaurant in Lubeck, Germany as a probable source of that outbreak. Tracing the epidemic revealed more than 3,800 cases, with HUS developing in more than 800 of the cases, including 36 fatal cases. Nearly $90 \%$ of the HUS cases were in adults (9).

Infected persons or healthy carriers transmit E. coli by their feces. E. coli contaminates the gastrointestinal tract of different animals - sheep, cows, goats. Food which is contaminated by their feces plays also a role as a factor of transmission $(2,3,4,6,8)$. Humans become infected by three different mechanisms: by direct contact with infected persons, animals and their excreta; by eating/drinking contaminated foods or drinks not well cleaned fruits /apples/, vegetables /lettuce/, uncooked meat /contaminated at slaughter/ or sea food, unpasteurized milk; drinking non-chlorinated water $(21,22,23)$. All objects in the encirclement of infected children such as contaminated toys, thermometers, pacifiers, dishes, underwear, towels, bed and so on are also factors of transmission (23).

D-HUS is a rare disease and accounts for no more than $10 \%$ of all cases. It affects all age groups, but the elderly predominantly. Its incidence in children is about one-tenth of that in D+HUS or 2 cases per 1000 000 persons annually (4). In Europe more than 1000 patients are reported with complement abnormalities resulting in 1.5-1.8 per million inhabitants (6). HUS which occurs before the age of 6 months is most often D-HUS, because less than $5 \%$ of D+HUS cases occur before the mentioned age $(9,10,21)$. There is no seasonal predilection.

As it was mentioned above, more than $90 \%$ of D+HUS cases in childhood are caused by infection with verotoxin producing E. coli or the so-called enterohaemorrhagic E. coli (VTEC, EHEC) and 70\% of them are associated with a 0157:H7 strain infection. Other serotypes that have the potential to cause D+HUS are 0111:H8, 0103:H2, 0121, 0145, 026, 0113, 0104:H4. Less frequently observed agents are Shigella dysenteriae type 1 or Citrobacter freundii. E. coli urinary tract infections cause that clinical syndrome rarely.Both variants of HUS cause endothelial damage, leukocyte activation, platelet activation, 
widespread inflammation and multiple thromboses in the small blood vessels - a condition known as systemic thrombotic microangiopathy (TMA). It leads to thrombotic events as well as organ damage/failure and death $(1,3,4)$. Because the pathogenesis of TTP is similar to the mentioned changes, all of these three conditions are included in the group of TMA (9).

EHEC produces two kinds of verotoxin: VT-1 and VT-2, which are known as Shiga-like toxins Stx-1 and Stx-2, because of its similarity to the toxin, produced by Shigella dysenteriae type I. Both kinds of verotoxin consist of two subunits: subunit $A$ and subunit $B$. Each Stx is encoded by a specific bacteriophage. Bacteriophages can be induced either spontaneously, or by some antibiotics, for example quinolones, which leads to increasing the level of Stx production and plays an important role in the pathogenesis of the disease as a trigger factor (10).

Subunit A of the verotoxin is responsible for cytotoxic effects and subunit $B$ is similar to membraneassociated glycosphingolipids: globotriaosylceramide (Gb3) and globotetraosylceramide (Gb4) mainly, but also to galabiosylceramide ( $\mathrm{Ga} 2$ ) and pentosylceramide (P1). Because of this, the organ distribution of the mentioned receptors determines the target zone of the patomorphologic changes - glomerular endothelium, brain and pancreas mainly $(3,4)$.

The causative agent enters the human body through the oral cavity by eating or drinking contaminated food or water. The colon is the first affected organ and the microorganism adheres to the epithelial cells in the terminal ileum and to the follicle-associated epithelium of Peyer's patches, followed by colonization of the colon $(8,10,13)$. Bacteria starts replicating here, produces Stx, which induce apoptosis of the affected cells and synthesis of proinflammatory cytokine such as IL-8. Inflammation is developed, which becomes increased by production of the endotoxin as well. It leads to acute diarrhea and hemorrhagic colitis occurs due to cell desquamation $(10,13,14)$.

Stx goes into the bloodstream by portocaval anastomosis which normally collects up to $6 \%$ of the blood which circulates in the gastrointestinal system (see Fig. 1). The presence of the Stx in the patients' blood leads to HUS or more rarely to thrombotic thrombocytopenic purpura (TTP). According to different studies, Stx is transported in the blood by binding to the Gb3 receptor of polymorphonuclear neutrophils (PMNs), erythrocytes, platelets or monocytes $(10,14,19,21)$. Neutrophils, monocytes and plasmocytes exhibit resistance to Stx's cytotoxic effect and because of this the toxin circulates in the bloodstream affecting target organs $(12,14,15,17,19,20)$.

The main pathogenetic mechanism of the HUS is endothelial dysfunction presented by thrombotic microangiopathy (TMA) in renal glomeruli, the gastrointestinal tract, brain and pancreas. During HUS, platelets are deposited on injured endothelial cells. Multiple microthrombi lead to thrombocytopenia. Platelets are activated by direct interaction with endotoxin, Stx and by factors released from damaged endothelium $(20,22,23)$. Together with thrombocytopenia, Stx induces elevation in the synthesis of tissue factor, which is the receptor for coagulation factor VII, thus converting factor $\mathrm{X}$ to $\mathrm{Xa}$ in the extrinsic pathway. Tissue factor expression will trigger thrombin generation resulting in clot formation and further platelet activation $(2,4,6,8)$.

The mechanism of hemolysis which occurs during HUS $(3,4,6)$ is still hotly debated. It is known that TMA includes changes of the vessel wall such as swelling or detachment of the endothelial cell from the basement membrane, accumulation of amorphous material in the subendothelial space, intraluminal 
thrombosis and partial or complete obstruction of the vessel lumen $(8,10,11)$. This suggests that microvascular endothelial cell injury is central to the pathogenesis of HUS. So hemolysis is probably the result of a mechanical breakdown in the occluded vessels $(19,20,21,22,23)$. According to other studies, oxidative stress is responsible for that process (18).

The main target organ affected in HUS is the kidney. In severe cases the brain may be involved, as well as other organs. Human kidneys exhibit both glomerular and tubular damage during HUS with extensive apoptosis of renal cortical cells (18). After binding to Gb3 on endothelial cells, Stx is internalized by receptor-mediated endocytosis (see Fig. 1). Inside the host cell, subunit A of the toxin is proteolytically cleaved to an enzymatically active fragment, which cleaves a residue within the 605 ribosomal subunit. This inhibits protein synthesis, causing cell death $(5,6,8,9,12,18,22)$. In addition to cytotoxicity, Stx may also exert activating effects on endothelial cells, for example the stimulation of interleukin-8 (IL-8) and monocyte chemoattractant protein-1 production and upregulation of adhesion molecule expression. Endothelin-1 is a potent vasoconstrictor produced by endothelial cells $(7,11)$. Stx causes upregulation of endothelin-1 (ET-1) mRNA and protein levels. Furthermore, Stx promotes leukocyte dependent inflammation and endothelial cell activation, with a change to a more procoagulant endothelial cell phenotype, in addition to endothelial cell damage, which will trigger platelet adhesion to the subendothelium.

Stx exerts a cytotoxic and apoptotic effect on glomerular endothelial and epithelial cells. The cytotoxic effect is enhanced in the presence of TNF- $\alpha 60$ as well as IL-1, LPS and butyrate. Studies have demonstrated that tubular cells are affected during HUS (18,23,28,31). Mesangial expansion, necrosis and mesangiolysis have been described in renal samples obtained during HUS, although some of the cases reported were presumably not EHEC associated. Mesangial cells also possess Gb3 receptors which are upregulated by TNF- $\alpha 124$ enabling Stx to inhibit protein synthesis and exert a cytotoxic effect, after prolonged incubation, as well as to reduce nitric oxide production $(4,6,8)$ Stx did not, however, stimulate the release of cytokines or chemokines from mesangial cells $(13,21)$.

The human brain expresses the Gb3 receptor in neurons and the endothelium $(4,6,8,9)$. Because of this some of the patients with HUS developed symptoms of the central nervous system varying form irritability to coma. 


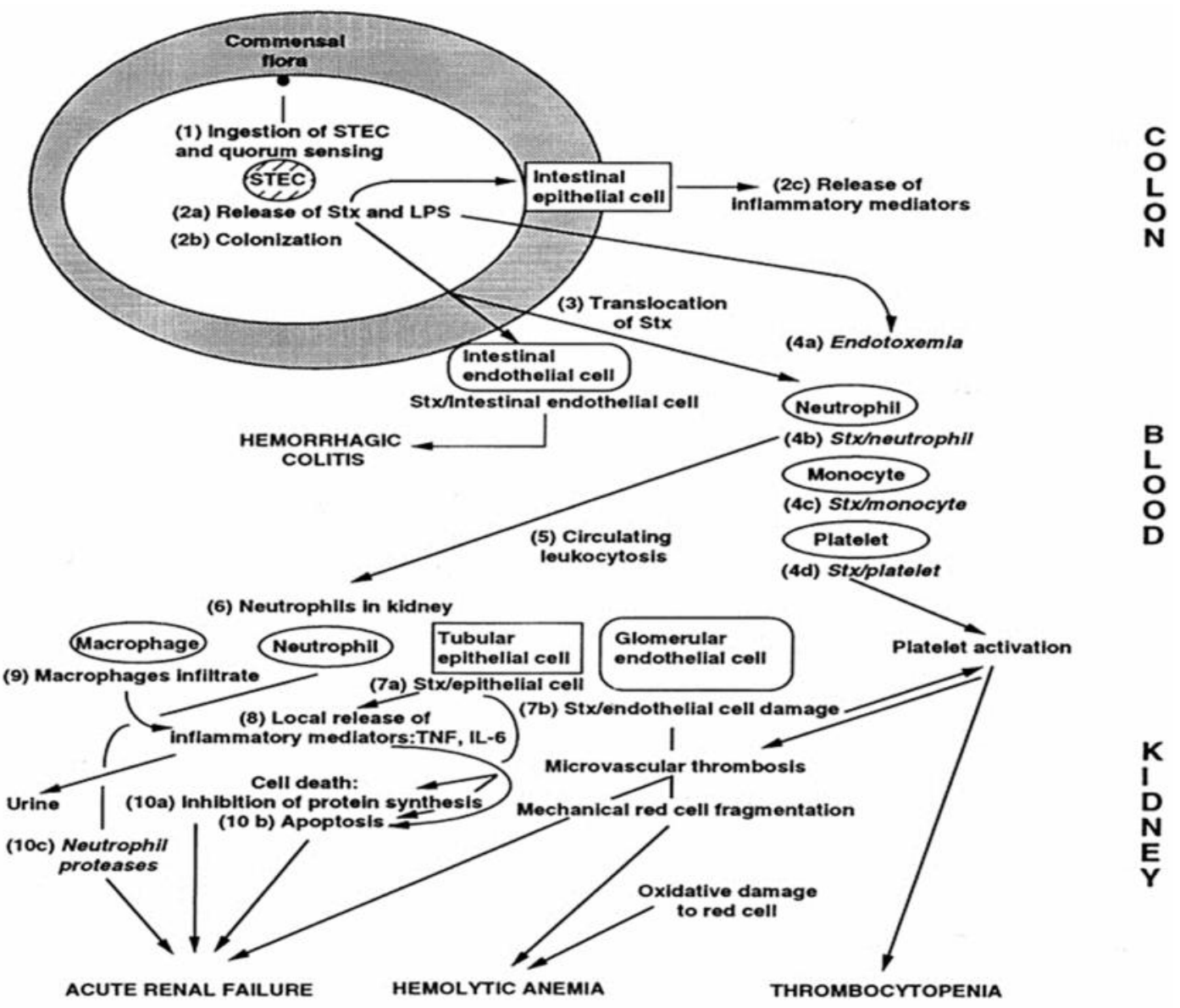

Figure 1 Pathogenesis of HUS, François Proulx, Ernest G Seidman and Diana Karpman, Pathogenesis of Shiga Toxin-Associated Hemolytic Uremic Syndrome, Pediatric Research (2001) 50, 163-171.

The disease starts acutely with symptoms as watery diarrhea (83\%) and abdominal cramps. Nausea and vomiting are also presented (30-60\%). In $70 \%$ of cases, diarrhea may be bloody and after a mean period of 6 days (2-14 days) from the onset of the diarrhea HUS begins $(1,3,6,8,9)$. The initial symptoms include worsening of the general condition of the patient, lethargy and irritability, acute severe pallor, and in some cases jaundice may develop. Fever is an accompanying symptom in 5-20\% $(13,21)$. The progression of the disease leads to decreased urine output (oliguria) (55\%), which is associated with edema formation, localized on the ankles predominantly. About $50-70 \%$ of the children with D+HUS develop acute kidney failure $(8,9,21)$. Malignant hypertension is presented in $47 \%$ of the cases and it is associated with kidney failure and central nervous system involvement $(21,22,23)$.

During the progression of the disease, extra renal symptoms develop. Most frequently the brain is involved, causing seizures $(20 \%)$, coma, cortical visual impairment, etc. $(2,3,6,8)$. Myocardial ischemia or abnormal heart rhythms are also typical signs. Pulmonary hemorrhages and pulmonary edema worsen the general condition of the patient in addition. 
Other extra renal manifestations include acute esophagitis, necrosis of the gut mucosa, intussusception, acute toxic hepatitis, and pancreatitis $(9,13,21)$.

The changes in the blood count include anemia mainly, which is presented by a fast decrease of the hemoglobin to $60-80 \mathrm{~g} / \mathrm{l}$ and in severe cases it can be decreased to $30-40 \mathrm{~g} / \mathrm{l}(1,6,8)$. Because the anemia is the microangiopathic hemolytic type, fragmented red blood cells (schistocytes, burr cells, helmet cells) can be seen in the blood smear, as a result of the destruction they cause when passing the damaged blood vessels (8). The grade of leukocyte count elevation is associated with the prognosis of the diseases and high leukocytosis indicates a poor outcome. Thrombocytopenia is moderate (from 25-55×109/l), sometimes transitory or relapsing and in $15-18 \%$ it is accompanied by skin petechial eruption without bleeding (8). Elevated lactate dehydrogenase LDH, decreased haptoglobin, and unconjugated hyperbilirubinemia indicate the breakdown of the red blood cells. Increase of the serum urea and creatinin shows the degree of the kidney failure. In the early stage of D+HUS, prothrombin time (PT) and activated partial thromboplastin time (APTT) are normal, which can be used to differentiate the disease form disseminated intravascular coagulation (DIC) $(3,6,8,9,13)$. In rare cases, only a decreased level of the antithrombin III and moderate fibrinolysis may be observed with elevation in the fibrin degradation products $(21,22)$. As an involvement of the other organs and system, elevation of the serum transaminases, pancreatic enzymes and blood glucose may be observed. Urinalysis reveals mild to moderate proteinuria, hematuria, or hemoglobinuria (see Fig. 2). During an ultrasound examination of the kidneys, they are enlarged, with higher echogenicity and Doppler ultrasound detects bloodstream disturbances in the kidneys' blood vessels (23).

The severity of the disease depends on some risk factors. The use of antibiotics such as quinolones, antimotillity agents, young age and female gender increase the risk for developing HUS after diarrhea. In the moderate forms, acute kidney failure is mild and the general condition of the patient is saved. Severe forms are presented by fever, leukocytosis, extra renal symptoms, anuria and hemodialysis is on demand $(1,6,8,9,10,16)$.

The normal course of the D+HUS prolongs 1-2 weeks and its outcome is marked by initially, an increase in the platelets count first, followed by normalization of the urine and an increase of the red blood cells. Hemoglobin becomes normal a month after the disease (23).

Patients with D-HUS have a history about preceding viral/bacterial infection, connective tissue disease or drug intake. An especially important feature is the anamnesis for family history. The disease starts secretly with atypical symptoms including hypertension and severe oligo-anuria $(3,6,8)$. In comparison with D+HUS, extra renal complications in patients with D-HUS are more common and more severe. Patients with a genetic form of HUS, as e result of ADAMTS 13 gene mutations, can present in infancy or later $(7,18,25)$. The evolution of the disease includes progression to ESRD. Laboratory changes vary from mild to severe hemolysis, jaundice and thrombocytopenia $(6,8,9,13,21,22,23)$.

When D+HUS is suspected a stool culture for E. coli and Shigella dysenteriae have to be collected and analyzed by cultural methods, polymerase chain reaction (PCR), serologic analyses and counting anti 0157 antibodies titers in the patient's sera. PCR and anti LPS antibodies should be conducted $(21,22,23)$. 
Shiga-toxin directly activates the alternative complement pathway and also interferes with complement regulation by binding to complement factor $\mathrm{H}$, an inhibitor of the complement cascade. Shiga-toxin causes complement-mediated platelet, leukocyte, and endothelial cell activation, resulting in systemic hemolysis, inflammation and thrombosis. Because of this C3 levels may be low in D+HUS but they are persistently low in D-HUS as a result of complement factor deficiency $(5,7,14,25)$.

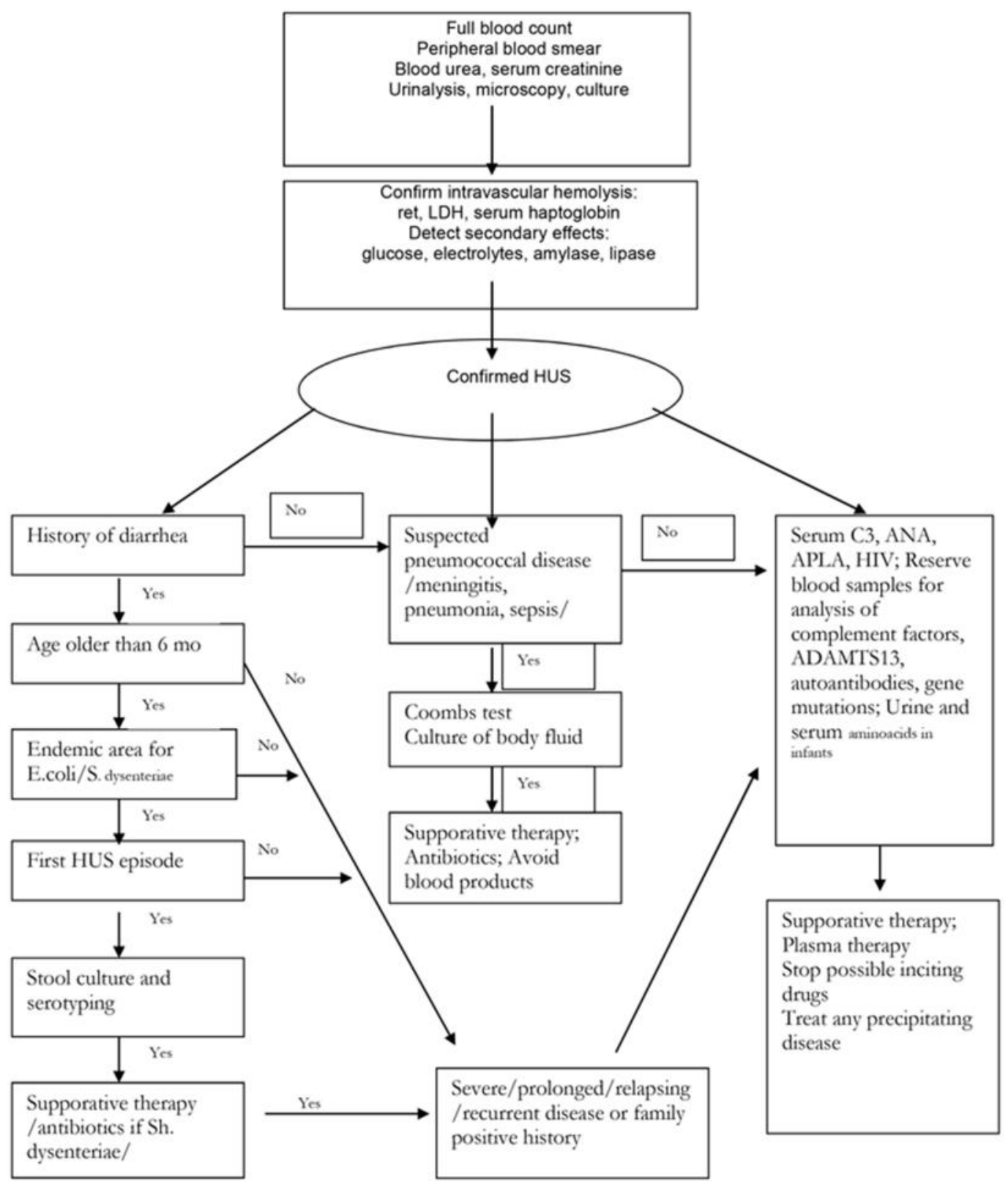

Figure 2 Diagnostic algorithm for patients suspected with HUS

In more than $90 \%$ of patients with pneumococcal HUS the direct Coombs test is positive $(36,37)$. 
When cobalamin metabolism defects are suspected, it is necessary to analyze the urine where homocystinuria is a persistent sign, together with hyperhomocystinemia, methyl malonic aciduria and low serum levels of methionine. Total serum vitamin B12 levels are normal $(25,28,33)$.

Autoimmune serology (ANA, antiphospholipid antibodies) and HIV screening may be indicated $(7,14,15,34)$.

In patients with no history of diarrhea, blood samples for the assay of specific complement factors (FH, $\mathrm{FI}$, MCP), and ADAMTS13 level should be collected, but it is genetic studies for mutations in complement factor genes, ADAMTS13 gene or cobalamin gene that provide definitive diagnosis $(5,7,14)$.

Because of the typical pathomorphologic changes in the affected organs, a renal biopsy is indicated to prove the diagnosis of HUS. But it is contraindicated in children and in the acute stage of the disease due to thrombocytopenia. It is indicated only when diagnosis is in doubt, or in recurrent or severe disease to confirm the diagnosis before starting aggressive therapy (21).

The characteristic pathologic findings of HUS are occlusive lesions of the arterioles and small arteries and consequent tissue microinfarctions (see Picture 1). In HUS, the lesions are usually limited to the kidneys, whereas the lesions are more widespread in TTP. Renal lesions are primarily focal and involve both the glomerular capillaries and the afferent arterioles. The venous side of the circulation is usually spared. A fully developed vascular lesion which consists of amorphous-appearing, hyalinelike, thrombi-containing platelet aggregates and a small amount of fibrin that partially or fully occludes the involved small vessels are typical for D+HUS. In D-HUS, thrombi consist of fibrin, together with platelet and VWF clumps involving larger renal and interlobular arterioles. Glomerular capillary wall thickening, occlusion or narrowing of capillary lumens, inflammation and necrosis of endothelial cells and their detachment from the basement membrane may be observed. Infiltration of macrophages and neutrophils are seen. Tubular epithelial injury, mesangial expansion and mesangiolysis may also be seen. Cortical necrosis is a rare sign associated with a severe form of the disease.

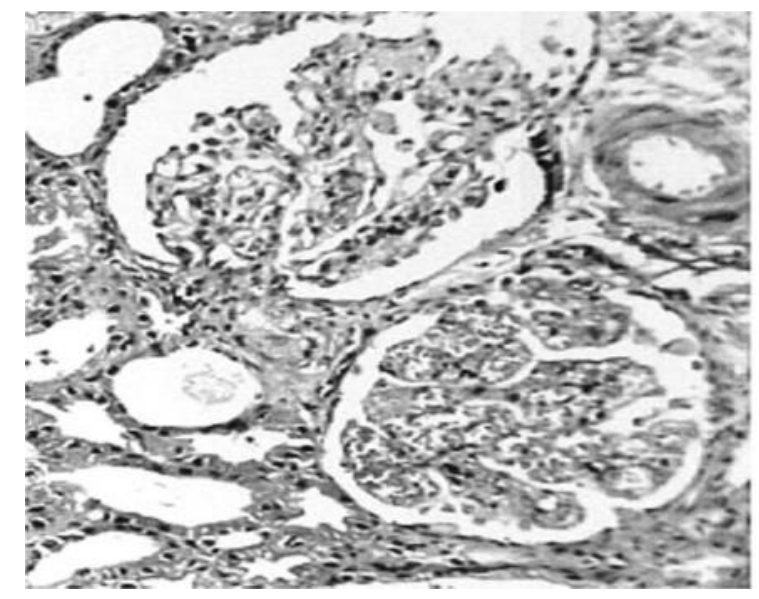

Picture 1. Renal biopsy of thrombotic microangiopathy in HUS, Banerjee Sushmita, Hemolytic uremic syndrome, Indian pediatrics 2009, 46, 1075-84. 
As it was mentioned above, TMA is a category of disorders that includes D+HUS, D-HUS and thrombotic thrombocytopenic purpura (TTP). Because of this the differential diagnosis among these three conditions is essential. All of them take their clinical course by decreasing the platelets count and microangiopathic hemolysis, and their clinical signs and symptoms may overlap: neurological symptoms (confusion, cerebral convulsions, seizures); renal impairment (elevated creatinine, decreased estimated glomerular filtration rate, abnormal urinalysis); gastrointestinal symptoms (diarrhea, nausea/vomiting, abdominal pain, gastroenteritis).

So when a patient is suspected for HUS, we have to determine the age first (aHUS affects children mainly), second - family history (positive in aHUS) and third - the initial symptoms of the disease (insidious with relapsing tendency in aHUS) (see Table 3).

If the patient experiences a first episode of HUS, which is preceded by acute diarrhea, especially bloody, and he/she is older than 6 months, D+HUS caused by Shiga-like toxin producing E. coli /Shigella dysenteriae should be considered. Because of the fact that $90 \%$ of HUS cases are D+HUS, a test for Shiga toxin/EHEC should be performed in all patients with HUS symptoms $(1,2,3,6,8,9,13)$.

But this is not a must because overlap syndromes exist - for example D+HUS may occur without diarrhea and aHUS as a result of complement abnormalities may take its clinical course in the presence of diarrhea. When the disease starts insidiously, there is a history for preceding diarrhea and tendency to relapse, affecting different family members, overlapping D-HUS should be considered and complement proteins and ADAMTS13 protease should be screened $(5,7,14,18,19,25)$.

Persistent complement abnormalities are typical for D-HUS. Decreased levels of C3 may be seen when mutations in CFH, CFI and MCP are presented (5). But normal levels of C3 do not exclude the diagnosis (5). In all cases of D-HUS levels of C4 stay normal. To prove the diagnosis D-HUS, all patients should be screened for $\mathrm{CFH}, \mathrm{CFI}$ and MCP mutations based on the frequency of mutations (CFH 30\%, MCP 10\% and CFI 2-5\%). Fluorescence-activated cell sorting (FACS) analysis is used to determine the MCP expression in leukocyte and this is needed in all patients suspected for aHUS together with measurement of anti factor $\mathrm{H}$ auto antibodies $(1,6,8,18,19,25)$. There is an exception for kidney transplanted patients where $\mathrm{MCI}$ mutations unlike MCP are associated with relapsing of the HUS in the transplanted kidney $(26,27,29,30)$. Because of this screening for factor I before MCP is essential for avoiding relapses after transplantation. Screening for complement factors should be performed in all patients with hemolysis, elevated liver enzymes, low platelets (HELLP) syndrome or post-partum or post-transplant HUS.

Complement mutations have been described in $36 \%$ of women with HELLP syndrome, $86 \%$ of cases of post-partum HUS and $29 \%$ of transplanted patients with de-novo posttransplant HUS $(21,22,23)$.

For this reason when a patient is suspected for aHUS and especially when neurologic symptoms appear, ADAMTS 13 activity should be measured (18). If its activity is lower than $10 \%$, screen for anti-ADAMTS13 antibodies. If the activity is persistently low without circulating antibodies, inherited deficiency is possible which suggests genetic analyses.

The features that mark defects in the cobalamin metabolism include early presentation of D-HUS in neonates or in infants under six-months of age. The first diagnostic step in this case is measurement of 
the homocystine and methylmalonate levels in urine, which are increased. After that genetic analyses may be implemented $(7,35)$.

Table 3 Differential diagnosis of HUS

\begin{tabular}{|c|c|c|}
\hline Age & Characteristic/Abnormalities & Diagnosis \\
\hline $0-6$ months & $\begin{array}{c}\text { Congenital complement abnormalities; } \\
\text { SAP-HUS; defects in cobalamine metabolism }\end{array}$ & $\begin{array}{c}\text { aHUS } \\
\text { DDx: SPA - HUS } \\
\text { Congenital TTP /Upshaw-Schulman } \\
\text { syndrome/ } \\
\text { Methylmalonic aciduria }\end{array}$ \\
\hline $\begin{array}{c}6 \text { months }-5 \\
\text { years }\end{array}$ & EHEC & $\begin{array}{c}\text { D+HUS } \\
\text { DDx: complement related HUS }\end{array}$ \\
\hline Adults & $\begin{array}{c}\text { Complement abnormalities }- \\
\text { MCP abnormalities or anti factor H antibodies }\end{array}$ & \begin{tabular}{c} 
DDx: TTP due to ADAMTS13 antibodies \\
\hline
\end{tabular} \\
\hline
\end{tabular}

Notice the use of drugs, transplantations or pregnancy as a potential cause for aHUS in adults. According to the consensus document published by Campistol et al., in patients with thrombocytopenia and microangiopathic hemolytic anemia, developing neurological symptoms and/or without renal involvement, and/or gastrointestinal involvement, ADAMTS 13 activity and Shiga toxin/EHEC tests should be evaluated first. If the ADAMTS13 activity is less than $5 \%$, TTP is the most likely diagnosis; if the Shiga toxin/EHEC test is positive, D+HUS is the most likely diagnosis. If the ADAMTS13 activity is higher than $5 \%$ and the STEC test is negative, a differential diagnosis must be distinguished between aHUS and secondary HUS.

Many other diseases are very similar to HUS and should be distinguished from it. Acute kidney failure may also be seen in the course of enteric infections as a result of severe dehydration, but absence of thrombocytopenia and hemolysis make that diagnosis impossible. The absence of the requisite changes in coagulation distinguishes HUS form DIC syndrome. Pancytopenia is typical for sepsis also but the clinical history will be different between these two diseases.

Patients with HUS should be treated in specialized nephrology centers or send to an intensive care unit if their general condition is severe. Frequent monitoring of electrolytes, blood pressure, hemoglobin, red blood cells, serum urea and creatinin and neurological and ventilator parameters should be implemented. The nutrition of the patients includes a high caloric diet with restrictions on salt intake.

Specific treatment depends on the type of HUS. The general measures are shown below.

The first line treatment in all patients is supportive, especially in the cases of $\mathrm{D}+\mathrm{HUS}$, aiming the prevention of diarrhea-associated dehydration and maintaining intravascular volume $(1,6,8)$. Using antidiarrheal drugs as antimotility agents has been associated with higher risk of developing HUS (16). When acute renal failure is presented, a limitation of intravenous fluids should be undertaken, and the use of diuretics, for example furosemide, ensures normal diuresis. In the cases when severe arterial hypertension is presented, an intravenous infusion with nitroprusside together with frequent monitoring of arterial blood pressure is recommended. If arterial hypertension persists in the next days, an oral antihypertensive drug should be administrated. If the hematocrit is lower than $20 \%(0.20 \mathrm{~g} / \mathrm{l})$ and the 
hemoglobin is lower than $60 \mathrm{~g} / \mathrm{l}$, a packed red blood cells transfusions is indicated. Platelets transfusions may deteriorate the general condition of the patient, causing further damage to the kidneys and because of this should be implemented only when active bleeding is presented or a surgical procedure is programmed (32).

About $50 \%$ of patients with D+HUS need early dialysis, especially if there is oliguria resistant to diuretics, worsening uremia (serum urea $>40 \mathrm{mmol} / \mathrm{l}$; serum creatinine $>600 \mathrm{mkmo} / \mathrm{l}$ ), thyperhydration, hyperkalemia $(>7 \mathrm{mmol} / \mathrm{l})$, hyponatremia, metabolic acidosis $(21,22,23,32)$.

Antibiotic treatment in D+HUS is controversial $(1,2,3,4,5,6,7,8,15,16,22,23,28,31,32,33)$. Some of the authors think that its use is associated with worsening of the clinical course of the disease, causing high production of verotoxin from dying bacteria. Antibiotics are indicated for the treatment of complications and the prevention of transmission if shigellosis is the cause for D+HUS. But in the cases of pneumococcal HUS, early antibiotic treatment is essential for saving a patient's life. Vancomycin is recommended, because of the high resistance to penicillin together with a third generation cephalosporin, and reassessment of the therapy is required when sensitivity results are ready $(24,26,37)$.

In aHUS early plasma therapy (24 hours after admission to the hospital) is essential, especially for TTP, as well as a first-line treatment until a normal platelets count, hemoglobin and LDH levels have been attained by plasma exchange and/or without FFP. Plasma exchange ( 1.5 plasma volumes per session five times a week for two weeks and three times a week for the subsequent two weeks) might be more effective than infusion, as it removes potentially toxic substances from the circulation but it is used together with fresh frozen plasma infusions more often because they avoid volume overload. Plasma exchange removes $\mathrm{CFH}, \mathrm{CFI}, \mathrm{CFB}$ and mutated $\mathrm{C} 3$ in addition to anti-factor $\mathrm{H}$-antibodies; an infusion of frozen fresh plasma (FFP) normalizes the levels of $\mathrm{CFH}, \mathrm{CFI}, \mathrm{CFB}$ and $\mathrm{C} 3$. Plasma exchange rather than infusion should be considered the first-line therapy in situations that limit the amount of plasma that can be infused, such as renal impairment or heart failure. Plasma treatment should be started within 24 hours of the patient's presentation, to decrease treatment failures. It should be continued once or twice a day for at least 2 days after complete remission. Plasma therapy is contraindicated in Streptococcus pneumoniaeinduced non-Stx-HUS; it may exacerbate the disease because adult plasma contains antibodies against the Thomsen-Friedenreich antigen. Some cases (HUS due to abnormal cobalamine metabolism or congenital ADAMTS13 deficit, or plasma resistant complement abnormalities) are resistant to plasma therapy and different treatment should be considered $(7,14,15,18,19,23,24,25,36,37)$.

The use of anticoagulants, such as heparins, steroids, antiplatelet and antioxidant drugs, is controversial. Intravenous immunoglobulin has been used in some studies in a daily dose of $2 \mathrm{ml} / \mathrm{kg}$, in a course duration of 2-5 days, leading to full recovery of the treated patients.

A new therapy has been developed nowadays using a drug SYNSORB Pk, which mediates the binding of Shiga toxin to endothelial cell surfaces and its sequestering in the intestine. The drug is still in the experimental phase $(21,22,23,28,31,35)$.

A new biologic therapy was implemented by Food and Drug Assistance (FDA) and the European Medicines Evaluation Agency (EMEA) using eculizumab for the cases of aHUS. Eculizumab is a recombinant, humanized monoclonal antibody against $\mathrm{C} 5$ that inhibits the activation of terminal components of complement. Its effect is associated with improvement in kidneys' function, including 
elimination of the need for dialysis in several cases that had not responded to plasma therapy. Patients treated with Eculizumab also exhibited improvement in platelet counts and other blood parameters. This drug is effective in D+HUS (used in the German outbreak in 2011) and severe TTP and ADAMTS 13 deficiency as a result of anti-ADAMTS 13 antibodies in patients who stay resistant to plasma therapy. Eculizumab is so effective in relapsing aHUS after renal transplantation $(21,22,23,35)$.

Because of the mechanism of Eculizumab action, the susceptibility to different infections becomes increased so treated patients should be vaccinated against Neisseria meningitidis, Haemophilus influenzae and streptococcus pneumoiniae mainly. Preventive antibiotic therapy should be also considered because lack of vaccination for some bacteria.

The duration of Eculizumab treatment is controversial and relapses have been registered after its interruption.

A monoclonal anti-CD-20 antibody - Rituximab has been evaluated in adults with unresponsive or relapsing TTP. Chemotherapy, as for example vincristine, cyclophosphamide and cyclosporine, has not been associated with better recovery.

The question of renal transplantation in patients with non-Stx-HUS is disputable because more than $50 \%$ of relapses have been registered. Relapse rates (30-100\%) are higher in patients with HF1 mutations than in those without this mutation. In patients with an MCP mutation, renal transplantation is associated with favorable outcomes because the missing protein is present in the cells of a transplanted kidney.

Transplantation is contraindicated if the donor is a family member because the familial genetic mutation risk is high. Renal transplantation can be safely performed in patients with $\mathrm{D}+\mathrm{HUS}(26,27,29,30)$.

In patients with $\mathrm{CHF}$ and $\mathrm{CFI}$ genetic defect, combined liver-kidney transplantation is considered because factors $\mathrm{H}$ and $\mathrm{I}$ are produced by the liver. Plasma therapy may improve the outcomes in these cases, when it is administrated before or after surgery.

The prognosis of HUS depends on its variant. The long term prognosis of $\mathrm{D}+\mathrm{HUS}$ is considerably more auspicious than that of other forms of HUS and up to $5 \%$ of children fail to regain renal function. The mortality rate in the course of appropriative therapy is about $5-15 \%$ as a result of extra renal complications mainly. In developing countries it is $70 \%$. But between $10-30 \%$ of patients develop chronic kidney disease and about $5-10 \%$ of these develop ESRD in the next 10 years $(1,2,3,4,6,8,9,13,21,22)$.

In streptococcal HUS, the prognosis depends on the severity of the present infection, and when meningitis occurs it is associated with up to $37 \%$ mortality rate $(36,37)$.

In non-infection related HUS, up to $25 \%$ of patients die in the acute phase of the disease and $50 \%$ progress to an ESRD. FH and FI disease is severe and the failure rate in renal transplantation in this case is high $-80 \%$ graft loss. MCP mutations have a good prognosis after renal transplantation. The prognosis of ADAMTS13 deficiency is favorable as a result of plasma therapy and the mortality rate is about $10-20 \%$ $(5,14,18,2325)$. 


\section{References}

1. Banatvala N, Griffin PM, Greene KD et al.: The United States National Prospective Hemolytic Uremic Syndrome Study: Microbiologic, serologic, clinical, and epidemiologic findings. J Infect Dis 183, 2001: $1063-1070$.

2. Bell BP, Goldoft M, Griffin PM et al. A multistate outbreak of Escherichia coli O157:H7-associated bloody diarrhea and hemolytic uremic syndrome from hamburgers. The Washington experience. JAMA 1994; 272: 1349-1353.

3. Besbas N, Karpman D, Landau D et al., A classification of hemolytic uremic syndrome and thrombotic thrombocytopenic purpura and related disorders. Kidney Int 2006; 70: 423-431.

4. Bhimma R, Rollins NC, Coovadia HM et al., Post-dysenterichemolytic uremic syndrome in children during an epidemicof Shigella dysentery in Kwazulu/Natal. Pediatr. Nephrol. 1997; 11: 560-564.

5. Blackal DPI, Marques M. Hemolytic uremic syndrome revisited-Shiga Toxin, factor $\mathrm{H}$ and fibrin generation. Am J Clin Pathol 2004; 121: S81-S88.

6. Boyce TG, Swerdlow DL, Griffin PM. Escherichia coli O157:H7and the hemolytic-uremic syndrome. N. Engl. J. Med. 1995; 333: 364-368.

7. Caprioli J, Noris M, Brioschi S et al. Genetics of HUS: the impact of MCP, CFH and IF mutations on clinical presentation, response to treatment, and outcome. Blood 2006; 108: 1267-1279.

8. Copelovitch L, Kaplan BS. The thrombotic microangiopathies. Pediatr Nephrol 2008; 23: 17611767.

9. Case definition for HUS-cases associated with the outbreak in Germany, WHO.

10. Elliott EJ, Robins-Browne RM, O'Loughlin EV et al. Nationwide study of haemolytic uraemic syndrome: Clinical, microbiological,and epidemiological features. Arch. Dis. Child. 2001; 85: 25131.

11. Griffin PM, Tauxe RV: The epidemiology of infections caused by Escherichia coli O157:H7, other enterohemorrhagic E. coli, and the associated hemolytic uremic syndrome. Epidemiol Rev 1991;13: 60-98.

12. Karch $\mathrm{H}$. The role of virulence factors in enterohemorrhagic Escherichia coli (EHEC) associated hemolytic-uremic syndrome. Semin Thromb Hemost 2001; 27: 207-213.

13. Karch H, Friedrich AW, Gerber A et al., New aspects in the pathogenesis of enteropathic hemolytic uremic syndrome. Semin Thromb Hemost 2006; 32: 105-112.

14. Karmali MA. Infection by Shiga toxin-producing Escherichia coli: an overview. Mol Biotechnol 2004; 26: 117-122. 
15. Kavanagh D, Richards A, Atkinson J. Complement regulatory genes and hemolytic uremic syndromes. Annu Rev Med 2008; 59: 293-309.

16. Kawasaki Y, Suzuki J, Nozawa R et al., A 12-year old girl with hemolytic uremic syndrome as initial symptom of systemiclupus erythematosus and a literature review. Am. J. Nephrol. 2002; 22: 576-80.

17. Locking ME, O'Brien SJ, Reilly WJ et al., Risk factors for sporadic cases of Escherichia coli 0157 infection: The importance of contact with animal excreta. Epidemiol Infect 127, 2001: 215-220.

18. Loirat C, Niaudet $\mathrm{P}$. The risk of recurrence of hemolytic uremic syndrome after renal transplantation in children. Pediatr Nephrol 2003; 18: 1095-1101.

19. Loirat C, Girma JP, Desconclois $C$ et al., Thrombotic thrombocytopenic purpura related to severe ADAMTS13 deficiency in children. Pediatr Nephrol 2009; 24:19-29.

20. Mannucci PM. Thrombotic thrombocytopenic purpura and the hemolytic uremic syndrome: much progress and many remaining issues. Haematologica 2007; 92: 878-880.

21. McCarthy TA, Barrett NL, Hadler J et al., Hemolytic-uremic syndrome and Escherichia coli 0121 at a lake in Connecticut, 1999. Pediatrics 108, 2001: E59.

22. Mead PS, Griffin PM: Escherichia coli O157:H7. Lancet 352,1998:1207-1212.

23. Meyers KE, Kaplan BS. Hemolytic uremic syndrome. In: Barratt TM, Avner ED, Harmon WE (eds). Pediatric Nephrology, 4th edn.Baltimore: Lippincott, Williams Wilkins, 1999; 811-22.

24. Michael M, Elliott EJ, Ridley GF et al., Interventions for hemolytic uraemic syndrome and thrombotic thrombocytopenic purpura. Cochrane Database Syst Rev 2009; 21: CD003595.

25. Nathanson S, Deschenes G. Pneumoniae-induced hemolytic uremic syndrome. Pediatr. Nephrol. $2001 ; 16: 362-365$.

26. Niaudet $\mathrm{P}$, Gagnadoux MF, Broyer $\mathrm{M}$ et al., Hemolytic-uremicsyndrome: Hereditary forms and forms associated with hereditarydiseases. Adv. Nephrol. Necker. Hosp. 2000; 30: 261-280.

27. Reynolds JC, Agodoa LY, Yuan CM et al., Thrombotic microangiopathy after renal transplantation in the United States. Am J Kidney Dis 42, 2003: 1058-1068.

28. Remuzzi G, Ruggenenti P, Colledan M et al. Hemolytic uremic syndrome: A fatal outcome after kidney and liver transplantation performed to correct factor $\mathrm{H}$ gene mutation. Am. J. Transplant.2005; 5: 1146-1150.

29. Richardson SE, Karmali MA, Becker LE et al., The histopathology of the hemolytic uremic syndrome associated with verocytotoxin-producing Escherichia coli infections. Hum. Pathol. 1988;19: 1102-1108.

30. Ruggenenti P: Post-transplant hemolytic-uremic syndrome. Kidney Int 62, 2002: 1093 -1104. 
31. Ruggenenti P, Noris M, Remuzzi G: Thrombotic microangiopathy, hemolytic uremic syndrome, and thrombotic thrombocytopenic purpura. Kidney Int 60, 2001: 831-846.

32. Safdar N, Said A, Gangnon RE et al., Risk of hemolytic uremic syndrome after antibiotic treatment of Escherichia coli O157:H7 enteritis. A meta-analysis. JAMA 2002; 288: 996-1000.

33. Scheiring J, Andreoli SP, ZimmerhackI LB. Treatment and outcome of shiga-toxin-associated hemolytic uremic syndrome. Pediatr Nephrol 2008; 23: 1749-1760.

34. Sharma AP, Greenberg CR, Prasad AN et al., Hemolytic uremic syndrome (HUS) secondary to cobalamin C (cblC) disorder. Pediatr Nephrol 2007; 22: 2097-2103.

35. Turner ME, Kher $\mathrm{K}$, Rakusan $\mathrm{T}$ et al. Atypical hemolytic uremicsyndrome in human immunodeficiency virus-1-infected children.Pediatr. Nephrol. 1997; 11: 161-3.

36. van de Kar NC, Roelofs HG, Muytjens HL et al. VerocytotoxinproducingEscherichia coli infection in hemolytic uremic syndromein part of western Europe. Eur. J. Pediatr. 1996; 155: 592-595.

37. Vanderkooi OG, Kellner JD, Wade AW et al. Invasive Streptococcus pneumoniae infection causing hemolytic uremic syndrome in children: Two recent cases Can J Infect Dis 2003; 14: 339343.

38. Waters AM, Kerecuk L, Luk $D$ et al., Hemolytic uremic syndrome associated with invasive pneumococcal disease: the United kingdom experience. J Pediatr 2007; 151: 140-144.

39. Zipfel PF, Skerka C: Complement factor $\mathrm{H}$ and related proteins: An expanding family of complement-regulatory proteins? Immunol Today 15, 1994: 121-126.

\section{Corresponding author}

Iliyan Todorov

Department of Infectious Diseases, Parasitology and Dermatology, Medical University of Varna

100, str Tzar Osvoboditel л. р свободител 100

Varna, 9000

Bulgaria

Phone: +359 883/310-376

e-mail: ilio_87@abv.bg 